\title{
Back to the drawing board-loss of chromosome $Y$ (LOY) in leukocytes is associated with age-related macular degeneration
}

\author{
Lars A. Forsberg $\mathbb{1 D}^{1,2,3}$
}

Received: 24 August 2018 / Accepted: 24 August 2018 / Published online: 23 October 2018

(c) European Society of Human Genetics 2018

Grassmann et al. [1]. show that mosaic loss of chromosome $\mathrm{Y}$ (LOY) in blood cells is associated with increased risk for late-stage age-related macular degeneration (AMD). This condition is a leading cause of vision impairment and blindness and affected about 6.2 million people globally in 2015 [2]. Previously established risk factors for AMD include age, inherited genetic variants as well as environmental factors such as smoking [1]. LOY is a during lifetime-acquired somatic mutation, leading to a mosaic state in which affected men harbors a percentage of cells without the $\mathrm{Y}$ chromosome. By studying LOY in blood samples from 5772 AMD cases and 6732 controls from 26 studies, Grassmann et al. are the first to show that LOY in blood is associated with increased risk for AMD (overall $\mathrm{OR}=1.33$ ), independent of other known confounders. Interestingly, analyses performed in different age groups suggest that the strength of the association between LOY and AMD was strongest in men 65-70 years of age (OR = 1.74), compared to younger and older age groups tested. Furthermore, they replicate previous results showing that age and smoking are important risk factors for LOY in peripheral blood.

A frequent loss of the $\mathrm{Y}$ chromosome in hematopoietic cells of men was first discovered more than 50 years ago, in the early days of cytogenetics [3]. It has since then been established that the frequency of LOY increases with age and that at least 10 and $20 \%$ of normally aging men around 70 and 80 years of age, respectively, harbor at least $10 \%$ of blood cells without a Y chromosome [4-7]. The data

Lars A. Forsberg

lars.forsberg@igp.uu.se

1 Department of Immunology, Genetics and Pathology, Uppsala University, Uppsala, Sweden

2 Science for Life Laboratory, Uppsala University, Uppsala, Sweden

3 Beijer Laboratory of Genome Research, Uppsala University, Uppsala, Sweden presented by Grassmann et al. validates these frequencies as $11 \%, 12 \%, 16 \%, 17 \%$, and $24 \%$ of the 12,504 studied men in the age groups $<65,65-70,70-75,75-80$, and $>80$ years of age, respectively, displayed LOY in blood. The corresponding numbers for the 6732 AMD free controls were $10 \%, 10 \%, 14 \%, 15 \%$, and $22 \%$, respectively. These results replicates previous results showing that LOY is the most frequent somatic human mutation and it is affecting $~ 1.6 \%$ of the genome [8]. In addition to age, smoking and genetic background have been described as significant risk factors to be affected with LOY in blood $[6,7,9]$. For example, in a study of 6014 men from three cohorts, Dumanski et al. first described that smoking was associated with LOY in a transient and dose dependent fashion and that current smokers have up to a four-fold risk for LOY in blood cells compared to never smokers [9]. Furthermore, by implementation of a Mendelian randomization approach, Wright et al. could infer a casual effect of smoking on LOY in blood in two cohorts (combined $n=18.508$, combined $p=$ 0.004) [7]. The association between smoking and LOY are replicated in the analysis presented by Grassmann et al., in both ever smokers $(\mathrm{OR}=1.69)$ and current smokers $(\mathrm{OR}=$ 2.64). In addition, a dose dependent effect from smoking on LOY in blood cells, first described by Dumanski et al. [9], is replicated $(\mathrm{OR}=1.01)$ [1]. Furthermore, genome-wide association studies have identified variation in several genomic regions that is associated with increased risk to carry blood cells with LOY, by studying germline inherited genetic variants and the level of LOY in participants of large cohorts [6, 7]. To date, 19 genomic loci have been identified to be associated with increased risk for LOY in blood, and these regions contain genes important for cell proliferation and cell cycle regulation [6, 7].

Mosaic LOY in blood was long considered to be a phenotypically neutral event and related to normal aging [10]. However, in 2014 a study demonstrated that LOY in peripheral blood cells is associated with all-cause mortality, using data from two independent prospective cohorts of aging men with up to 20 years follow-up time [4]. It was 
found that elderly men with LOY in $>35 \%$ of the blood cells at study entry survived on average only half as long as the age-matched controls. It was also described that men with LOY in leukocytes had an increased risk to develop and die from tumors outside the hematological system [4]. Subsequent studies of specific cancer types have validated that cancer patients on average have higher levels of LOY in the blood cells compared to controls in colorectal and prostate cancer [11], bladder and prostate cancer [6], and testicular germ cell tumors (TGCT) [12]. Interestingly, the men studied in the latter study had a median age close to 30 years of age, suggesting that LOY in blood is an important risk factor for cancer development also in young men. In addition to being associated with increased risk for various forms of non-hematological cancers, men with LOY in leukocytes have been found to have an increased risk for other disorders and outcomes such as Alzheimer's disease [5], autoimmune conditions [13, 14], myelodysplastic syndrome [15], suicide completion [16], as well as major cardiovascular events [17]. The present results from Grassmann et al., adds AMD to this growing list of human disorders that are associated with LOY in blood cells.

A critical question to address in future studies is how LOY in leukocytes could be connected with increased risks for disease in tissues and organs outside the hematological system. Since the increased risk for disease mediated by age have been fitted in the statistical models in the abovementioned papers, it is not likely that co-variation between disease incidence and level of LOY in older men, would explain the observations. Furthermore, the TGCT-study [12] and the Grassmann et al. study demonstrates that the increased risk for disease mediated by LOY in blood is not exclusive for elderly men. Another hypothesis that could help explain the associations between LOY in blood and risk for disease in other organs, is that LOY in blood cells might cause disturbances to vital immune system functions, such as immunosurveillance and regulation of inflammation, which is normally performed by leukocytes in blood [4, 5, 8, 9, 18]. Accordingly, LOY-dependent dysregulation of disease-protective immune functions could lead to an increased risk for disease development such as neoplastic proliferation of cells in various organs leading to cancer; increased neurodegenerative processes in the central nervous system associated with development of AD; as well as increased risk of atherosclerotic plaques and major cardiovascular events. Of note, genome-wide association studies has identified the complement cascade system to be consistently involved in AMD [1]. A possible connection between this result and the association between AMD and LOY in blood cells needs to be further studied. Nevertheless, the results in Grassmann et al. can be viewed as support for the hypothesis that LOY in leukocytes is an important factor for the immune system's ability to combat disease processes taking place in different tissues and organs.

Men in the entire world live shorter lives compared to women and the global difference is about 4 years. This sex bias is larger in populations with longer life expectancy, for example, about 7 years in Japan [18]. The results from recent studies showing that LOY in leukocytes are associated with the major causes of death, could help explain the shorter lifespan of men, since LOY is a male specific risk factor. Clinical detection of men with LOY have the potential to identify men with increased risk for disease and thus, facilitate earlier diagnoses as well as enabling more personalized treatment options. Further research of LOY and its consequences will also increase our understanding of disease mechanisms and possibly the development of new preventive treatments in the field of precision medicine.

Acknowledgements This work was supported by grants from the European Research Council ERC Starting Grant, the Swedish Research Council, Stiftelsen Olle Engkvist Byggmästare, and Kjell och Märta Beijers Stiftelse to L.A.F.

\section{Compliance with ethical standards}

Conflict of interest L.A.F. is co-founder and shareholder in Cray Innovation $\mathrm{AB}$.

\section{References}

1. Grassmann F, Kiel C, den Hollander AI, et al. Y chromosome mosaicism is associated with age-related macular degeneration, Eur J Hum Genet. 2018. https://doi.org/10.1038/s41431-0180238-8.

2. Global Burden of Disease Study 2013 Collaborators. Global, regional, and national incidence, prevalence, and years lived with disability for 301 acute and chronic diseases and injuries in 188 countries, 1990-2013: a systematic analysis for the Global Burden of Disease Study 2013. Lancet . 2015;386:743-800.

3. Jacobs PA, Brunton M, Court Brown WM, Doll R, Goldstein H. Change of human chromosome count distribution with age: evidence for a sex differences. Nature . 1963;197:1080-1.

4. Forsberg LA, Rasi C, Malmqvist N, et al. Mosaic loss of chromosome $\mathrm{Y}$ in peripheral blood is associated with shorter survival and higher risk of cancer. Nat Genet. 2014;46:624-8.

5. Dumanski JP, Lambert JC, Rasi C, et al. Mosaic loss of chromosome $\mathrm{Y}$ in blood is associated with alzheimer disease. Am J Hum Genet. 2016;98:1208-19.

6. Zhou W, Machiela MJ, Freedman ND, et al. Mosaic loss of chromosome $\mathrm{Y}$ is associated with common variation near TCL1A. Nat Genet. 2016;48:563-8.

7. Wright DJ, Day FR, Kerrison ND, et al. Genetic variants associated with mosaic Y chromosome loss highlight cell cycle genes and overlap with cancer susceptibility. Nat Genet. 2017;49:674-9.

8. Forsberg LA, Gisselsson D, Dumanski JP. Mosaicism in health and disease-clones picking up speed. Nat Rev Genet. 2017; 18:128-42. 
9. Dumanski JP, Rasi C, Lonn M, et al. Mutagenesis. Smoking is associated with mosaic loss of chromosome Y. Science. 2015; 347:81-83.

10. United Kingdom Cancer Cytogenetics Group. Loss of the $\mathrm{Y}$ chromosome from normal and neoplastic bone marrows. Genes Chromosom Cancer. 1992;5:83-8.

11. Noveski P, Madjunkova S, Sukarova Stefanovska E, et al. Loss of $\mathrm{Y}$ chromosome in peripheral blood of colorectal and prostate cancer patients. PLoS ONE. 2016;11:e0146264.

12. Machiela MJ, Dagnall CL, Pathak A, et al. Mosaic chromosome Y loss and testicular germ cell tumor risk. J Hum Genet. 2017; 62:637-40.

13. Persani L, Bonomi $\mathrm{M}$, Lleo $\mathrm{A}$, et al. Increased loss of the $\mathrm{Y}$ chromosome in peripheral blood cells in male patients with autoimmune thyroiditis. J Autoimmun. 2012;38: J193-6.
14. Lleo A, Oertelt-Prigione S, Bianchi I, et al. Y chromosome loss in male patients with primary biliary cirrhosis. J Autoimmun. 2013;41:87-91.

15. Ganster C, Kampfe D, Jung K, et al. New data shed light on Yloss-related pathogenesis in myelodysplastic syndromes. Genes Chromosomes Cancer. 2015;54:717-24.

16. Kimura A, Hishimoto A, Otsuka I, et al. Loss of chromosome $\mathrm{Y}$ in blood, but not in brain, of suicide completers. PLoS ONE. 2018;13:e0190667.

17. Haitjema S, Kofink D, van Setten J, et al. Loss of Y chromosome in blood is associated with major cardiovascular events during follow-up in men after carotid endarterectomy. Circ Cardiovasc Genet. 2017;10:e001544.

18. Forsberg LA. Loss of chromosome Y (LOY) in blood cells is associated with increased risk for disease and mortality in aging men. Hum Genet. 2017;136:657-63. 